\title{
Tratamientos psicológicos
}

\author{
MARTín, L.J.
}

Psicólogo. Dirección General de Atención a las Drogodependencias. Gobierno de Canarias

\section{RESUMEN}

El tratamiento del alcoholismo tiene un importante componente psicosocial, del cual los tratamientos psicológicos constituyen el núcleo esencial. El abordaje psicológico debe tomar en consideración en primer término la motivación del paciente, y en esta línea se revisan abordajes motivacionales estructurados como las intervenciones motivacionales breves, las entrevistas motivacionales y las terapias de mejora motivacional.

Desde los modelos cognitivo-conductuales también se han desarrollado tratamientos centrados en la mejora de las habilidades de afrontamiento, en la potenciación de las habilidades sociales y en la prevención de recaídas. Asimismo, desde los modelos de condicionamiento clásicos, también se han diseñado intervenciones basadas en la exposición a estímulos.

La terapia de grupo aparece como uno de los tratamientos de elección, existiendo en nuestro país modelos de intervención profesionalizados, que van más allá de los grupos de autoayuda. Finalmente, se hace un especial hincapié en la importancia de la familia en el tratamiento del alcoholismo y se presenta el Modelo de Reforzamiento Comunitario (MRC) como un acercamiento integrador.

Palabras clave: tratamiento, psicoterapia, habilidades sociales, psicoterapia de grupo, alcoholismo, familia.

\begin{abstract}
The treatment of alcoholism has an important psychosocial component, whose essential nucleus is constituted by the psychological treatments. The psychological approach must, in the first place, take into consideration the motivation of the patient, and motivational approaches structured as brief motivational interventions, motivational interviews and improved motivational therapies are reviewed from this perspective.

Treatment has also been developed from the cognitive-behavioural models, focused on improvement in confrontation abilities, on promoting social abilities and on the prevention of relapses. In addition, interventions have been designed from classical conditioning models, based on exposure to stimuli.

Group therapy appears as one of the available treatments, with the existence of models of professional intervention in our country that go beyond self-help groups. Lastly, there is a special emphasis on the importance of the family in the treatment of alcoholism and the Community Reinforcement Model (CRM) is presented as an integrator approach.
\end{abstract}

Key words: treatment, psychotherapies, social skills, group psychotherapy, alcoholism, family.

\section{INTRODUCCIÓN}

tratamiento psicológico de la dependencia alcohólica ha estado, y en cierta manera, sigue estando, enormemente condicionado por multitud de factores de tipo social, clínico e incluso moral. Es quizás en el tratamiento de la dependencia alcohólica donde nos encontramos, a lo largo de los años, con una mayor cantidad de "tratamien- 
tos" de dudosa utilidad clínica y de discutible ética profesional.

Sin embargo, a lo largo de los años, el tratamiento de los problemas derivados del consumo abusivo y de la dependencia alcohólica ha experimentado un notable desarrollo y un no menos importante apoyo científico al trabajo clínico.

En las próximas paginas nos proponemos exponer los abordajes psicoterapéuticos de la dependencia alcohólica describiendo aquellos modelos, técnicas y estrategias más utilizadas en al actualidad y que cuentan con un apoyo clínico y empírico contrastado.

Como señalan Di Clemente et al. [1] la motivación es un factor crucial para emprender un camino de cambio conductual. Si un individuo no quiere cambiar su estilo de vida nosotros no podremos forzarle a hacerlo. Este argumento se vuelve especialmente relevante en el caso de la adicción al alcohol. La motivación del individuo a abandonar la conducta de consumo de alcohol se convierte en el factor más importante a la hora de comenzar y mantener un camino hacia el cambio. Hasta hace relativamente poco tiempo los profesionales "esperaban" a que la motivación del individuo "apareciese" y éste se decidiera a abandonar la bebida abusiva, ya que se consideraba inútil intentar cualquier tratamiento si el paciente no estaba suficientemente motivado.

Durante los últimos años, sin embargo, los clínicos e investigadores han mostrado un renovado interés por la motivación para el cambio y, especialmente por el proceso de cambio de conducta. El modelo transteórico de estadios de cambio [2] proporcionó un marco teórico adecuado para la comprensión de proceso de cambio en la conducta adictiva como un fenómeno activo, influenciable por el sujeto y su entorno y enormemente ligado a la motivación del individuo para cambiar. Los estadios de cambio propuestos en el modelo transteórico bajaron la última barrera (hasta la fecha) de la comprensión del fenómeno de recuperación de la adicción cuyo precedente anterior lo encontramos en el modelo de prevención de recaídas de Marlatt y Gordon [3] que explica la importancia de la recaída en la recuperación del paciente alcohólico y los factores que influyen en ella.

La motivación es una dimensión crítica en la influencia que tiene sobre el paciente para comenzar el tratamiento, comprometerse con él y completarlo, así como realizar cambios a largo plazo en su conducta de beber. Los estudios realizados con pacientes adictos han demostrado la importancia de la motivación para el tratamiento para predecir la participación en el tratamiento y la recuperación [4]. Los modelos tradicionales han utilizado estrategias agresivas o confrontacionales para afrontar la falta de motivación del paciente [5]. En un tipo de procedimiento ampliamente utilizado, un grupo de miembros de la familia se reúnen para confrontar al paciente y convencerle de que el tratamiento de su alcoholismo es necesario [6]. Las evidencias recientes indican, sin embargo, que la confrontación puede fortalecer la negativa del paciente y su resistencia al tratamiento [7], sugiriendo el tratamiento de los pacientes no motivados con estrategias generadoras de motivación y no con estrategias de confrontación.

Las fuentes de motivación para el cambio (intrínsecas o extrínsecas) se han comparado por diversos autores a fin de constatar cuales son las que aseguran un éxito mayor y más duradero en el tratamiento. Generalmente las fuentes internas de motivación se han asociado con un cambio a largo plazo [8] de forma más consistente que las fuentes externas. La severidad de los problemas de alcohol del paciente incrementa la motivación interna, presumiblemente porque la severidad del problema incrementa la angustia influenciando así la toma de decisiones. Aunque la motivación interna parece ser más efectiva para un éxito a largo plazo, la motivación externa y los incentivos parecen promover la abstinencia a corto plazo [10]. 


\section{MODELOS MOTIVACIONALES DE TRA- TAMIENTO}

\subsection{Intervenciones Motivacionales Breves}

La intervención motivacional breve (una sesión) utiliza consejo directo e información sobre las consecuencias negativas del abuso de alcohol para motivar a los pacientes para reducir la bebida o dejar de beber. Este tipo de intervención es más efectiva con bebedores problemáticos que no son adictos al alcohol que con pacientes dependientes del alcohol [11]. El objetivo de la intervención breve es más la reducción de la bebida que la abstinencia. Los terapeutas aconsejan al paciente acerca de la necesidad de reducir su consumo de alcohol y ofrecen feedback sobre los efectos de las conducta de beber del paciente. Este tipo de intervención parece contribuir, en gran manera a modificaciones en los patrones de consumo de los bebedores excesivos y siempre muy condicionado por el grado de motivación que tenga el individuo hacia el cambio de conducta [11].

\subsection{Entrevista Motivacional}

Este modelo desarrollado por Miller \& Rollnick [5] asume que el paciente es responsable de cambiar su conducta adictiva y reconoce la ambivalencia como parte del proceso. En contraste con los tratamientos confrontacionales, la Entrevista Motivacional (EM) está diseñada para ayudar a los pacientes a trabajar con su ambivalencia y evolucionar hacia un cambio positivo de la conducta.

El terapeuta utiliza varias técnicas para ayudar al paciente a incrementar la motivación para cambiar su conducta. Una técnica es la escucha reflexiva, una forma de parafraseo que permite al paciente contar su historia y sentir que está siendo escuchado por un terapeuta empático. Una segunda técnica incluye explorar los pros y los contras del cambio, lo que puede ayudar al paciente a evaluar de forma realista su conducta e, idealmente, determinar que los pros superan a los con- tras. Una tercera técnica, que apoya la autoeficacia del paciente, o la confianza en que puede cambiar, puede ayudarle a superar la barrera entre el deseo del paciente de cambiar y el cambio efectivo. Una cuarta técnica utiliza la entrevista y la evaluación para proporcionar al paciente feedback personalizado acerca de su conducta problema. Otra de las técnicas propone emitir frases automotivantes, tales como el reconocimiento del problema o la preocupación por la propia calidad de vida. En definitiva el terapeuta enfatiza la importancia de la elección personal del paciente respecto al cambio de conducta, restando importancia a las clasificaciones diagnósticas y evita discutir o confrontar al paciente, buscando que la confrontación aparezca intensamente como consecuencia de la percepción de las discrepancias internas.

\subsection{Terapia de Mejora Motivacional}

Este modelo específicamente diseñado para el proyecto MATCH por Miller y colaboradores combina las técnicas EM con la brevedad de una intervención menos intensiva. La Terapia de Mejora Motivacional (TMM) está compuesto por cuatro sesiones de tratamiento precedidas de una evaluación intensiva.

En la primera sesión el terapeuta proporciona al paciente un feedback claro, estructurado y personalizado acerca de su frecuencia de bebida (numero de días que ha bebido por mes), intensidad de la bebida (número de bebidas por sesión), nivel típico de intoxicación, riesgo de consecuencias negativas del uso de alcohol, resultados en la función hepática y pruebas neuropsicológicas, y factores de riesgo en relación al alcohol (v.g. riesgo familiar y síntomas de tolerancia).

En la segunda sesión, el terapeuta se concentra en el fortalecimiento del compromiso del paciente para cambiar utilizando técnicas que son apropiadas para el estadio de cambio en que se encuentra el paciente.

Durante la tercera y cuarta sesión el terapeuta se centra en revisar los aspectos ambivalentes que aún permanecen en el paciente 
para renovar la motivación y el compromiso de cambio. Por último en la cuarta sesión se discuten los planes de futuro del paciente.

\section{MODELOS CONDUCTUALES DETRATA- MIENTO}

\subsection{Terapia Cognitivo-Conductual de Habi- lidades de Afrontamiento [12]}

El término Terapia Cognitivo-Conductual de Habilidades de Afrontamiento (TCCHA) se refiere a un conjunto de herramientas terapéuticas para el tratamiento de la dependencia alcohólica y de otros desordenes psiquiátricos cuyo objetivo es tratar al paciente mejorando sus habilidades cognitivas y conductuales, cambiando sus conductas problemáticas. Este modelo conceptúa cualquier tipo de psicopatología, incluyendo la dependencia alcohólica, como un proceso maladaptativo de aprendizaje. Así, el objetivo central de los tratamientos TCCHA es diseñar técnicas a través de las cuales las respuestas maladaptativas puedan ser "desaprendidas" y reemplazadas con conductas adaptativas.

Durante los últimos 25 años se han desarrollado multitud de modalidades de TCCHA para el tratamiento del alcoholismo. Estos acercamientos han diferido en muchos sentidos incluyendo duración, modalidad, contenido y lugar de tratamiento [7]. A pesar de sus diferencias, sin embargo; todas las modalidades de TCCHA comparten dos elementos centrales.

- Abrazan los principios de la teoría sociocognitiva de Bandura [13] aplicados a la dependencia alcohólica, proclamando un papel central de las habilidades de afrontamiento [14].

- Emplean alguna forma de entrenamiento individual en estrategias de afrontamiento para mejorar los deficits del paciente. Para mejorar las habilidades de afrontamiento del paciente en esas situaciones todos las variantes de TCCHA utilizan herramientas de entrenamiento tales como instrucciones, modelado, juego de roles y ensayo conductual.

TCCHA se clasifica frecuentemente como un "modelo de tratamiento de amplio espectro", esto es, un acercamiento que no se enfoca solo a los problemas de alcohol del paciente sino que además trabaja con otros problemas y áreas asociados generalmente a la conducta de beber y a la recaída.

Un elemento clave de TCCHA es el análisis detallado del patrón de consumo de cada paciente para identificar los antecedentes individuales y consecuentes de la conducta de beber. Los resultados de este análisis funcional proporciona la base para desarrollar un plan específico de tratamiento para el paciente. Dicho plan puede indicar qué situaciones debe evitar el paciente, cómo debe manejar el paciente dichas situaciones si tienen lugar, y qué conductas alternativas (distintas a la bebida) debe usar el paciente para afrontar dichas situaciones.

Las últimas investigaciones en el campo de la efectividad de la TCCHA demuestran claramente que la efectividad de la misma puede incrementarse sustancialmente. Una forma de llevar a cabo esto sería incorporar componentes de otras terapias efectivas, así como otras terapias han ampliado su enfoque y aumentado su efectividad incluyendo estrategias TCCHA.

En primer lugar, se ha encontrado que la entrevista motivacional - una estrategia dirigida a incrementar la motivación del paciente para el cambio - incrementa la efectividad de otro tipo de terapias [15].

En segundo lugar la investigación ha mostrado consistentemente que la implicación del paciente en grupos de autoayuda, tales como Alcohólicos Anónimos, se asocia con resultados positivos de bebida y potencia la efectividad de TCCHA, particularmente si incluye grupos de autoayuda con sistemas de creencias similares a aquellos subyacentes a la TCCHA (v.g. SMART Recovery [16]). En SMART la asunción subyacente es que el alcohol es, al menos en parte, una conducta maladaptativa 
y que la persona por si misma tiene la capacidad de producir un cambio en la misma.

En tercer lugar, muchos estudios han demostrado la efectividad de los modelos terapéuticos que tratan de incluir una o más personas relevantes en el tratamiento de personas con problemas de alcohol tales como Terapia de Parejas o Reforzamiento Comunitario. La incorporación de factores ambientales en la TCCHA también puede incrementar el control terapéutico sobre los factores reforzantes que pueden ayudar a un paciente a mantener un estilo de vida libre de alcohol.

En cuarto lugar, la TCCHA puede fácilmente incorporar estrategias de tratamiento basadas en procedimientos de condicionamiento clásico tales como exposición a estímulos [17].

En quinto lugar aunque las actuales TCCHA se enfocan generalmente a entrenar habilidades para afrontar las situaciones con un gran riesgo de recaída, los primeros estudios sobre la efectividad de la TCCHA incluían frecuentemente el entrenamiento en habilidades sociales generales.

\section{EL ENTRENAMIENTO EN HABILIDADES DE AFRONTAMIENTO [17]}

El Entrenamiento en Habilidades de Afrontamiento (EHA) tiene como objetivo aumentar las habilidades de afrontamiento del paciente y proporcionarle estrategias específicas para afrontar la necesidad de beber. Los investigadores hipotetizan que, como resultado de la práctica de habilidades de afrontamiento, los pacientes se sentirán menos distorsionados por las situaciones de riesgo y esto facilitará el que no se produzca una recaída después del tratamiento.

De acuerdo a la teoría del aprendizaje social, existen multitud de factores que pueden incrementar la posibilidad de que un alcohólico recaiga cuando se le confronta con una situación estresante o con otra situación que está asociada con una alta posibilidad de beber.
El entrenamiento en habilidades está diseñado para enfrentarse a los mencionados factores en multitud de formas. En primer lugar los terapeutas pueden entrenar a los clientes en la utilización de habilidades específicas para determinadas situaciones de riesgo, para aumentar la capacidad del mismo para afrontar dichas situaciones en el futuro. En segundo lugar los terapeutas pueden entrenar a los clientes en habilidades para mejorar las relaciones sociales estando sobrio y para reducir los conflictos tanto en la familia como en el trabajo. En tercer lugar y como resultado de la ampliación de las habilidades sociales y de afrontamiento, se generará una mayor expectativa de autoeficacia, lo que redundará positivamente en la utilización de dichas habilidades en situaciones de alto riesgo.

Son muchas las evidencias científicas que sustentan la importancia del entrenamiento en habilidades para los pacientes alcohólicos. Estos estudios indican, en primer lugar, que las habilidades de afrontamiento de los alcohólicos son menores que las de los no alcohólicos para afrontar determinadas situaciones, particularmente aquellas en las que está implicada una recaída [17].

La teoría del aprendizaje social sugiere asimismo que multitud de factores de tipo personal o ambiental y situaciones determinadas colocan a un bebedor en riesgo determinado de recaída. Un entendimiento de dichas situaciones puede ayudar a los terapeutas a diseñar entrenamientos de habilidades de afrontamiento específicas que puedan necesitar los pacientes en situaciones determinadas.

Por lo general todos los acercamientos de EHA comienzan con una evaluación de las áreas de vulnerabilidad del paciente [17]. Esta evaluación se puede centrar en muchos aspectos, tales como condiciones biológicas o psiquiátricas coexistentes, factores de riesgo intrapersonales e interpersonales y la cantidad de exposición que el paciente va a tener a estímulos relacionados con la bebida. El terapeuta deberá evaluar los riesgos interpersonales del paciente, incluyendo las habilidades sociales generales, las habilidades de rechazo, así como el tipo de situaciones inter- 
personales que han contribuido en el pasado a una pauta de ingesta alcohólica excesiva. Una manera muy útil de conseguir un registro detallado de éstas es proponerle al propio paciente un auto registro diario de las mismas. Esto puede ayudar al paciente y al terapeuta a identificar tanto los factores de riesgo como los protectores. Dos de las estrategias más utilizados de EHA son la prevención de recaídas y el entrenamiento en habilidades sociales o de comunicación.

\subsection{Prevención de recaídas}

Cada sesión se enfoca a una situación específica de riesgo de recaída y se enseñan varias habilidades para manejar dicha situación.

Este acercamiento, incluye típicamente ocho sesiones, cada una de las cuales se centra en un tipo de situación que presenta una alta posibilidad de recaída. Estas situaciones se clasifican en cuatro grandes categorías (1) frustración y enfado (2) tentaciones interpersonales (ofrecimiento de una bebida) (3) estados emocionales negativos y (4) tentaciones intrapersonales. Estas diversas situaciones así como las estrategias adecuadas para manejarlas se encuentran descritas por Marlatt y Gordon [3]. La prevención de recaídas se puede desarrollar en terapia individual o en terapia de grupo incluyendo cada sesión los siguientes componentes.

- Instrucciones directas de estrategias de afrontamiento para situaciones específicas.

- Modelado de dichas situaciones por los terapeutas o por los miembros del grupo.

- Ensayo de las conductas en juego de roles.

- Retroalimentación acerca de las respuestas de los pacientes y de lo que los pacientes piensan durante estas situaciones ensayadas.

- Instrucciones acerca del proceso cognitivo para generar las respuestas alternativas a las situaciones de riesgo.
A través de este proceso el paciente aprende a resolver las situaciones que se le puedan presentar en la vida real teniendo la oportunidad de discutir todos los pasos de forma pormenorizada y desmenuzando los más pequeños aspectos que se puedan generar. Este tipo de entrenamiento desarrollado en grupo presenta la ventaja de proporcionar una enorme riqueza de situaciones diferentes que al tratamiento individual pueden escapársele y generar alternativas desde la propia experiencia vicaria de un paciente incluido en la terapia.

\subsection{Entrenamiento en habilidades sociales o de comunicación.}

En la que cada sesión se enfoca en una habilidad interpersonal general diseñada para mejorar las relaciones interpersonales. Esta aproximación de tratamiento trata de reducir conflictos, mejorar los apoyos sobrios del alcohólico y cambiar su estilo de vida.

Al contrario de la prevención de recaídas, el entrenamiento en habilidades de comunicación se centra en las habilidades de comunicación que pueden utilizarse para manejar una variedad de situaciones de riesgo, más que en las habilidades específicas para manejar un determinada situación de riesgo [17].

Este acercamiento incluye las siguientes habilidades: (1) rehusar una bebida, (2) proporcionar feedback positivo, (3) proporcionar criticas de forma efectiva, (4) recibir críticas acerca del uso del alcohol, (5) desarrollar habilidades de escucha, (6) mejorar las habilidades de conversación, (7) desarrollar apoyos para la sobriedad y (8) aprender formas efectivas de resolución de conflictos.

Asimismo, los autores proponen el desarrollo de otras cinco habilidades adicionales si el diseño del grupo y el tiempo lo permite: (1) aspectos no verbales de la comunicación, (2) expresión de sentimientos (3), introducción a la asertividad (4), saber decir no y (5) manejo de la crítica en general.

Este tipo de modalidad de tratamiento se desarrolla generalmente en grupo. Cada sesión consta de un resumen de los objeti- 
vos de la misma y de porque es importante para la sobriedad seguido de una pequeña discusión de la habilidad a desarrollar. Los pacientes deberán generar una ejemplos personales de los posibles escenarios en los cuales dicha habilidad puede ser utilizada. Como en la prevención de recaídas, las sesiones incluyen entrenamiento en habilidades de afrontamiento efectivas, modelado de dichas habilidades por parte del terapeuta y de los miembros del grupo, role playing y análisis de la respuesta y de los aspectos cognitivos de la misma.

\section{TRATAMIENTO CON EXPOSICIÓN A ES- TÍMULOS}

Los modelos de condicionamiento clásico de indicadores de alcohol y recaída sugieren que los estímulos ambientales que en el pasado estaban asociados a la bebida pueden generar respuestas condicionadas que pueden jugar un papel importante en la precipitación de una recaída. Las evidencias actuales [18] sugieren que las respuestas inducidas por estímulos se asemejan más a respuestas apetitivas condicionadas. La teoría del aprendizaje social sugiere que la presencia de estímulos puede incrementar el riesgo de recaída incrementando la relevancia de los efectos positivos del alcohol para el bebedor, lo que puede empujar al alcohólico a beber más alcohol. El Tratamiento con Exposición a Estímulos (TAE) puede ejercer sus efectos beneficiosos a través de dos mecanismos diferentes:

- En primer lugar, la teoría del aprendizaje establece que la exposición repetida a un estímulo (la visión de la bebida) al mismo tiempo que previene la respuesta habitual (la bebida) debe resultar en la disminución de las reacciones a medida que las sesiones avanzan (habituación) hasta una posible pérdida permanente de la respuesta elicitada (salivación o ansiedad).

- En segundo lugar, la teoría del aprendizaje social sugiere que implicarse en la práctica de habilidades de afrontamiento debe incrementar tanto la efectividad de dichas habilidades como la creencia del alcohólico acerca de su habilidad para responder diligentemente cuando se enfrente con indicadores similares en la vida real. Como resultado de esta práctica repetida las reacciones internas a los indicadores de alcohol interferirán menos con la habilidad del bebedor para utilizar sus habilidades de afrontamiento en el futuro.

\section{TERAPIA FAMILIAR}

El alcoholismo está relacionado con infinidad de trastornos relacionales inlcuyendo la violencia familiar, paternidad/maternidad inadecuada, disfunciones sexuales, discordia doméstica en general, así como aumento de las tasas de divorcio. El alcoholismo implica, asimismo, una pesada carga financiera para los alcohólicos y sus familias debido, principalmente a la inestabilidad laboral y los problemas legales causados por la adicción de uno o más miembros de la familia [19].

Los estudios clínicos y de investigación indican que los problemas familiares y maritales no solo pueden precipitar la bebida abusiva, sino que pueden mantener un patrón de bebida excesiva una vez que ésta se ha desarrollado así como romper la sobriedad en alcohólicos abstinentes [19]. Los investigadores han encontrado altos niveles de conflictividad, negatividad y competitividad, y niveles menores que la media de expresividad, cohesión y habilidades para la resolución de conflictos en las familias alcoholicas [19].

Los enfoques tradicionales han separado a la familia del tratamiento del alcohólico [20]. El tratamiento orientado desde un modelo de enfermedad familiar generalmente trata a los alcohólicos individualmente, mientras que el tratamiento de los miembros de la familia se desarrolla en la educación acerca del alcoholismo y los programas de Doce Pasos orien- 
tados específicamente a miembros de la familia del alcohólico.

\section{TERAPIA CONDUCTUAL DE PAREJAS}

La Terapia Conductual de Parejas (TCP) trabaja directamente para incrementar los factores de relación que conducen a la abstinencia [21]. Un modelo conductual asume que los miembros de la familia pueden recompensar la abstinencia, y que los pacientes alcohólicos con relaciones más felices y cohesionadas y con mejor comunicación tienen un riesgo menor de recaída. El paciente y su cónyuge acuden a 15-20 sesiones ambulatorias de TCP durante unos cinco o seis meses.

Las intervenciones conductuales incluyen un contrato de sobriedad diario. Esto implica una discusión de confianza diaria en la cual el alcohólico confirma su deseo de permanecer abstinente ese día y la esposa le agradece su compromiso. Para aquellos pacientes que están tomando Antabus (disulfiram), existe un ritual diario en el que los alcohólicos agradecen a su esposa que les vean tomar la dosis diaria de la medicación anti-bebida, mientras que la esposa se lo agradece al alcohólico y registra la acción en el contrato diario establecido previamente. Ambos cónyuges acuerdan no discutir cuestiones relacionadas con la conducta alcohólica en el pasado y reservarlo para las sesiones de terapia. Al comienzo de cada sesión de TCP en parejas se revisa el registro del contrato de sobriedad para comprobar el grado de cumplimiento de los mismos. Este registro proporciona un seguimiento continuado del progreso de la terapia que es recompensado verbalmente cada sesión. La Prevención de Recaídas es la actividad final de la TCP.

Utilizando una serie de técnicas conductuales, la TCP incrementa los sentimientos positivos, actividades en común y la comunicación constructiva ya que estos factores de relación incrementan la sobriedad [21].

Quizás el modelo mejor conocido de Terapia Conductual de Parejas (TCP) en el trata- miento del alcoholismo es el Proyecto de Asesoramiento para Matrimonios Alcohólicos (CALM) [21], un programa conducido por el Departamento de Psiquiatría de la Escuela de Medicina de Harvard en el Centro de la Administración de Veteranos de Brockton y en otras áreas de Boston. El programa, que empezó en 1978, tiene una simple premisa; las esposas pueden recompensar la abstinencia del alcohólico debido a que una mejor comunicación y una mayor cohesión familiar juegan un papel esencial en el proceso de recuperación. El resultado es un modelo de tratamiento que maneja simultáneamente la bebida abusiva y los problemas maritales combinando tratamiento individual, en parejas y grupal en parejas con un fuerte componente conductual.

\section{TERAPIA DE GRUPO CON ALCOHÓLI- COS [22]}

La Terapia de Grupo es una herramienta terapéutica utilizada ampliamente en el tratamiento del alcoholismo con diferentes tipos de programas y procedimientos. Entre estos están, Alcohólicos Anónimos, grupos de familias, grupos educacionales, psicodrama, grupos introspectivos, y grupos motivacionales. Aunque los terapeutas y pacientes que han participado en terapia de grupo la consideran útil y eficiente, la carencia de evidencia científica de sus mecanismos de acción ha conducido a una situación donde las opiniones son pasionales pero no basadas empíricamente. Muchos estudios han señalado lo difícil que es evaluar los procesos de terapia de grupo y su eficacia. Asimismo la comparación entre terapia individual y terapia de grupo es complicada y los resultados permanecen poco claros. Los estudios que evalúan la terapia de grupo han mostrado que los pacientes que están en psicoterapia grupal mejoran su grado de compromiso, tienen tasas más altas de abstinencia mejoran en un número de variables relacionadas con la calidad de vida, mejoran su transición de tratamiento interno 
a tratamiento ambulatorio y son más proclives a volver a las citas de seguimiento. Debe apuntarse, sin embargo, que la extrapolación de estos resultados es difícil, ya que se han obtenido con diferentes tipos de orientaciones, objetivos, técnicas y liderazgos.

Lo mecanismos subyacentes a la psicoterapia de grupo permanecen desconocidos. ¿Se debe a factores simplemente como acudir, presión de grupo, identificación, procesos de aprendizaje o todos ellos conjuntamente. Cuestiones acerca de la duración del tratamiento, liderazgo, estilo, número y tipo de tipo de pacientes y las condiciones de facilitación de la participación no son fáciles de contestar. El final de la terapia ha sido relacionado estrechamente a la recaída.

Un estudio [22] confirma, tras el examen y seguimiento de 329 alcohólicos en terapia de grupo la utilidad de esta herramienta terapéutica en el tratamiento de la enfermedad en la que se señala como crítica la adherencia al tratamiento como principal predictor del éxito del mismo. Los mismos autores $[23,24]$ han confirmado recientemente la efectividad de las técnicas grupales en un ensayo aleatorizado, y la persistencia de sus efectos a largo plazo.

\section{EL MODELO DE REFORZAMIENTO CO- MUNITARIO}

La filosofía subyacente al Modelo de Reforzamiento Comunitario (MRC) [25] es enormemente sencilla: para solucionar los problemas de alcohol es muy importante reorganizar la vida del individuo de manera que la abstinencia resulte más reforzante que la bebida. Este modelo se presenta como una alternativa integradora y que, de alguna forma, recoge las herramientas terapéuticas y modelos recientes de tratamiento del consumo abusivo y de la dependencia alcohólica desde una perspectiva global.

Los modelos tradicionales para dejar de beber han consistido, principalmente en aumentar el dolor, para confrontar a la persona con las consecuencias negativas de la bebida de una forma exagerada. Esta aproximación o modelo de tratamiento trata de convertir la bebida en algo poco atractivo y puede incluir terapias aversivas, terapias farmacológicas con disulfiram, counselling de confrontación o infligir consecuencias negativas (castigos). Estos acercamientos, sin embargo, se han mostrado inoperantes a la hora de disminuir la ingesta de alcohol o de tratar el alcoholismo [23].

EI MRC presenta un acercamiento diferente al problema del alcoholismo, que está basado en proporcionar incentivos para interrumpir la bebida más que administrar castigo por beber. EI MRC tiene dos objetivos principales: la eliminación de las consecuencias positivas de la bebida y el aumento del refuerzo positivo de la sobriedad. Para obtener estos resultados los terapeutas MRC deben combinar una serie de estrategias de tratamiento: motivación, análisis de los patrones de consumo, incremento de refuerzos positivos, entrenamiento de conductas e implicación de personas significativas.

\section{A) Construcción de la motivación}

Como inicio debemos explorar la motivación del individuo para cambiar. Esta evaluación debe hacerse con un estilo de entrevista motivacionalmente empátíco más que con estilo confrontativo [5], animando al cliente, más que al terapeuta a verbalizar las ventajas del cambio y las desventajas de la conducta de beber actual.

\section{B) Iniciando la sobriedad}

Una vez que el cliente ha identificado los factores que pueden motivarle a cambiar su conducta de beber, el terapeuta establece los objetivos para obtener la abstinencia. El muestreo de bebida, entre otros puede ser un procedimiento adecuado para establecer estos objetivos. Este procedimiento utiliza varias estrategias de counselling para negociar metas intermedias tales como un periodo de prueba de sobriedad. 


\section{C) Analizando los patrones de consumo de alcohol}

EI MRC implica un análisis funcional de los patrones de bebida del cliente. Este paso que se enfatiza muy poco en la terapia cognitivo conductual, resulta muy útil para individualizar el tratamiento y para determinar los componentes específicos, o módulos, que pueden ser más exitosos para un determinado paciente.

\section{D) Incremento del refuerzo positivo}

Tanto el cliente como el terapeuta deben seleccionar módulos apropiados de un menú de procedimientos de tratamiento adaptados a las necesidades específicas del cliente. Muchos de estos módulos se basan en incrementar las fuentes de reforzamiento positivo no relacionadas con la bebida que posee el cliente. Una de las formas más habituales es revertir el proceso de aislamiento del cliente alcohólico integrándolo en el grupos de no bebedores e incrementando el rango de actividades atractivas que no suponen beber. Estos planes enfatizan las actividades que ponen al cliente en contacto con otras personas en contextos no bebedores. La elección de dichos programas debe estar adaptado a las preferencias del cliente para evitar incorporarlo a actividades que realmente no le apetecen. Asimismo el cliente tiene la necesidad de ser asesorado en las cuestiones relativas a la vida diaria tales como aprender a comprar en un supermercado, comprar el periódico o utilización del teléfono. No debemos olvidar que el paciente ha abandonado un entorno seguro (la bebida) para incorporarse en un mundo, en cierta manera, desconocido, y que el peor enemigo puede ser el desanimo y la sensación de aislamiento en este nuevo contexto.

El objetivo común de estos módulos de tratamiento es conseguir que la vida sobria del cliente resulte más reforzante que la anterior afirmando y reincorporando al mismo en su comunidad.

\section{D) Entrenamiento de conductas}

Los pacientes practican las nuevas conductas y estrategias de afrontamiento durante las sesiones de tratamiento, mediante, por ejemplo modelos de juego de roles en sesiones individuales o grupales.

\section{E) Implicación de personas significativas}

La cooperación de otras personas que están cercanas o son significativas para el cliente resulta crucial para el éxito del tratamiento. Las personas significativas, particularmente aquellas que viven con el consumidor, pueden ser muy útiles en identificar el contexto social del cliente cuando bebe y en consecuencia, proporcionar apoyo para cambiar tal conducta.

Se han identificado diversos factores que inciden directamente en la efectividad del MRC. Entre ellos mencionaremos el estilo del terapeuta y la intensidad inicial del tratamiento.

\section{A) Estilo del terapeuta}

Un terapeuta MRC satisfactorio debe ser consistentemente positivo, enérgico, optimista y entusiasta. Cualquier progreso del paciente sin tener en cuenta lo pequeño que sea se debe reconocer y premiar en alguna manera. MRC se desarrolla en un entorno personal y en cierta forma, comprometido, no de forma automática o como si se tratase de un negocio. Las características de personalidad y de entrenamiento previo del terapeuta se pueden convertir en importantes aliados para poder llevar a cabo de forma exitosa una MRC.

\section{B) Intensidad inicial del tratamiento}

Otra característica importante de MRC es la intensidad inicial del tratamiento y la calidad del comienzo del mismo. Si un paciente esta preparado para el cambio de conducta y solicita una primera cita, ésta debe proporcionársele lo mas pronto posible, de forma ideal 
el mismo día o al día siguiente. Asimismo, durante la primera fase del tratamiento las sesiones de consejo deben llevarse a cabo, probablemente, más de una vez a la semana.

Finalmente MRC puede incluir procedimientos terapéuticos para iniciar la abstinencia inmediatamente, tales como iniciar una pauta de disulfiram. Para ello debemos intentar que el paciente y aquellas personas que le están apoyando en el tratamiento aseguren una correcta toma de la medicación y un seguimiento adecuado de las pauta terapéuticas.

MRC también ha sido integrado en un tratamiento familiar unilateral (TF) donde la persona que solicita tratamiento no es el bebedor que rechaza integrarse en un tratamiento) sino su esposa o un familiar preocupado por el problema. Sin la presencia del bebedor el terapeuta trabaja con la familia para cambiar el entrono cercano del bebedor poco a poco, retirando refuerzos de la bebida e incorporando refuerzos a la abstinencia o no bebida. El terapeuta también entrena a la familia para cuando el bebedor muestre de nuevo interés en comenzar un tratamiento.

\section{CONCLUSIÓN}

Aunque en la actualidad disponemos de múltiples modelos de intervención y de diversas técnicas que han probado su eficacia en condiciones experimentales, a los clínicos nos queda la asignatura pendiente de identificar qué tratamientos serán más eficaces en qué pacientes. En este sentido, los avances son todavía escasos y, a los menguados resultados obtenidos en el Proyecto Match [26], cabe añadir la poca utilidad clínica de las diversas tipologias del alcoholismo existentes, a la hora de decidir cual es el tratamiento de elección en un determinado paciente. El criterio clínico sigue siendo pues el elemento esencial en la elección del tratamiento más conveniente.

\section{BIBLIOGRAFÍA}

(1) DICLEMENTE, C.C., BELLINO, L.E. Y NEVINS, T.M. Motivation for Change an Alcoholism
Treatment. Alcohol Research \& Health. 23(2), 1999.

(2) PROCHASKA, J.O.; DICLEMENTE, C.C.; AND NORCROSS, J.C. In search of how people change: Applications to addictive behaviors. American Psychologist 47(9), 1992.

(3) MARLATT, G.A.; AND GORDON, J.R., EDS. Relapse Prevention: Maintenance Strategies in the Treatment of Addictive Behaviors. New York: Guilford Press, 1985.

(4) DELEON, G.; MELNICK, G.; AND KRESSEL, D. Motivation and readiness for therapeutic community treatment among cocaine and other drug abusers. American Journal of Drug \& Alcohol Abuse 23, 1997.

(5) MILLER, W.R., AND ROLLNICK, S. Motivational Interviewing: Preparing People to Change Addictive Behavior. New York: Guilford Press, 1991.

(6) LIEPMAN, M.R. Using family influence to motivate alcoholics to enter treatment: The Johnson Institute Intervention Approach. In: O'Farrell, T.J., ed. Treating Alcohol Problems: Marital and Family Interventions. New York: Guilford Press, 1993.

(7) MILLER, W. R.; BENEFIELD, R. G.; AND TONIGAN, J.S. Enhancing motivation for change in problem drinking: A controlled comparison of two therapist styles. Journal of Consulting and Clinical Psychology 61(3) 1993.

(8) DECl, E. L., AND RYAN, R. M. Intrinsic Motivation and Self-Determination in Human Behavior. New York: Plenum Press, 1985.

(9) HIGGINS, S.T., AND BUDNEY, A.J. Treatment of cocaine dependence through the principles of behavior analysis and behavioral pharmacology. In: Onken, L.S.; Blaine, J.D.; and Boren, J.J., eds. Behavioral Treatments for Drug Abuse and Dependence. NIDA Research Monograph No. 137. Rockville, MD: National Institute on Drug Abuse, 1993.

(10) HEATHER, N. Interpreting the evidence on brief interventions for excessive drinkers: The need for caution. Alcohol and Alcoholism 30(3), 1995.

(11) SPIVAK, K.; SANCHEZ-CRAIG, M.; AND DAVI$L A, R$. Assisting problem drinkers to change on their own: Effect of specific and non-specific advice. Addiction 89(9), 1994.

(12) LONGABAUGH, R. \& MORGENSTERN, J.. Cognitive-Behavioral Coping-Skills Therapy fpr 
Alcohol Dependence. Alcohol Research \& Health, 1999.

(13) BANDURA, A. Social Foundations of Thought and Action. Englewood Cliffs, NJ: Prentice Hall, 1986.

(14) ABRAMS, D.B., AND NIAURA, R.S. Social learning theory. In: Blanke, H.T., and Leonard, K.E., eds. Psychological Theories of Drinking and Alcoholism. New York: Guilford Press, 1987.

(15) BROWN, J.M., AND MILLER, W.R. Impact of motivational interviewing on participation in residential alcoholism treatment. Psychology of Addictive Behaviors 7, 1993.

(16) SMART Recovery News and Views. Vol. 1, 1995.

(17) MONTI, P.M.; ROHSENOW, D.J. Coping-Skills Training and cue-Exposure Therapy in the Treatment of Alcoholism. Alcohol Research \& Health. 23(2), 1999.

(18) NIAURA, R.S.; ROSENHOW, D.J.; BINKOFF, J.A.; MONTI, P.M.; ABRAMS, D.A.; AND PEDRAZA, M. The relevance of cue reactivity to understanding alcohol and smoking relapse. Journal of Abnormal Psychology 97, 1988.

(19) Rotunda R, O'Farrell T (1997), Marital and family therapy of alcohol use disorders: bridging the gap between research and practice. Professional Psychology: Research and Practice 28(3):246-252
(20) Steinglass P (1994), Family Therapy: alcohol. In: Textbook of Substance Abuse, Galanter M, Kleber HD, eds. Washington, D.C.: Psychiatric Press, pp 315-329.

(21) O'FARRELL, T.J. Behavioral Couples Therapy for Alcoholism and Drug Abuse Psychiatric Times, Vol. XVI (4) 1999

(22) MONRAS, M. \& GUAL, A. Attrition in Group Therapy with alcoholics: a Survival Analysis. Drug and Alcohl Review, 19, 2000.

(23) Monras M, Freixa N, Ortega L, Lligoña A, Mondon S, Gual A. Eficacia de la terapia de grupo para alcohólicos. Resultados de un ensayo clinico controlado. Medicina Clínica 2000: 116; 126-131.

(24) Lligoña A, Costa S, Gual A, Monrás M. 'Efficacité de la therapie de groupe chez le patient alcoolodépendant' Alcoologie et addictologie 2000: 22(4): 401-407.

(25) MEYERS, R.J., AND MILLER, W.R., EDS. A Community Reinforcement Approach to Addiction Treatment. Cambridge, UK: Cambridge University Press, 1999.

(26) Project Match Research Group (1997) Matching Alcoholism Treatments to Client Heterogeneity: Project MATCH posttreatment drinking outcomes. Journal of Studies on Alcohol 58 (1) pp 7-29. 